\title{
CVaR in Portfolio Optimization: An Essay on the French Market
}

\author{
Houda Hafsa ${ }^{1}$ \\ ${ }^{1}$ University of Carthage, Tunisia; Aix en Provence University, France \\ Correspondence: Houda Hafsa, Aix en Provence University, France. E-mail: houdahafsa@yahoo.fr
}

Received: January 23, 2015

Accepted: March 26, 2015

Online Published: April 9, 2015

doi:10.5430/ijfr.v6n2p101

URL: http://dx.doi.org/10.5430/ijfr.v6n2p101

\begin{abstract}
There has been a growing interest in CVaR as a financial risk measure in optimal allocation fields. This interest is based many key advantages of CVaR over the most used measures of risk: the Value-at-Risk and the variance. In this paper we develop an asset allocation model that allocates assets by minimizing CVaR subject to a desired expected return and we compare the performance of the resulting optimal portfolios with those resulting from the optimization of mean-variance model. The empirical study uses stocks from the SBF250 index. The purpose of the paper is to highlight the influence of the non-normal characteristics of the return distribution on the optimal asset allocation and test the superiority of the mean- CVaR approach over the mean-variance approach.
\end{abstract}

Keywords: portfolio optimization, $\mathrm{VaR}, \mathrm{CVaR}$, portfolio performance, non- normal distributions

\section{Introduction}

In today's increasingly turbulence and volatility on every major stock exchange, it is evident that controlling the risks in one's investment strategies is an important issue. The portfolio optimization problems have been one of the important research fields in modern risk management. In generally, a rational investor always prefers to have the return on his portfolio as large as possible. At the same time, he also wants to make the risk as small as possible. However, a high return always accompanied with a higher risk. The seminal work of Markowitz (1952) demonstrated that financial decision-making is essentially a question of achieving an optimal trade-off between return and risk measured by variance.

The mean-variance model has been one of the most commonly adopted models in asset allocation field. The main idea underlying this model is that variance of the return distribution is all what we need to describe the risk of the portfolio. Few years after, Markowitz (1959) highlighted several drawbacks, the most important being: that the variance of a portfolio penalizes the positive variation and negative variation in the same way; and that is not a suitable measure to capture infrequent events having low probability of occurrence as it is presumed by the kurtosis measure. Theoretically the model works only when the return distribution is multivariate normal or the utility of the investor is quadratic function of the return. Or it is widely recognized that neither the first nor the second assumption holds in practice and the extreme loss must be considered to describe adequately the distribution of returns. When investors do not have quadratic utility and returns are not normally distributed, variance is no longer an appropriate measure of risk since it ignores the higher moments of the return distribution.

A new field of interest has emerged with the introduction of the notion of value at risk (VaR) by JP Morgan American Bank in the beginning in 1996, filling the large gap in the financial industry's need to assess the extreme risks associated with the most of investments. The central idea behind the use of VaR is to summarize into a single number all the information about the possible portfolio losses implied by the left hand side tail of the return distribution in the case when this distribution is not normal. VaR is defined as the maximum expected loss on an investment over a specified horizon given some confidence level. VaR has quickly become universally adopted measure of risk (Note 1). The use of VaR has become the reference in risk management, such that controlling authorities have imposed regulatory constraints on the asset allocations of financial institutions based on the estimation of VaR. However, Artzner $(1997,1999)$ laid out the desirable mathematical properties that a risk measure has to possess in order to reflect the commonly accepted behavior of rational investors. Artzner and al. (1999) have defined the conditions for a coherent risk measures "and unfortunately VaR does not satisfy all of them. In fact, VaR lacks the property of subadditivity; therefore, it does not provide the investors with an incentive to diversify their investment 


\section{Literature Review}

Therefore, there has been growing interest in Conditional Value-at-Risk (CVaR) as a financial risk measure to improve the VaR computationally and theoretically. The CVaR measure introduced by Rockafellar and Uryasev $(2000,2002)$ the expected loss in the left tail given (i.e. conditional on) a particular threshold that has been met, such as the worst $1 \mathrm{st}, 5$ th or $10^{\text {th }}$ percentile of the distribution of possible future outcomes, it quantifies losses exceeding $\mathrm{VaR}$ and acts as an upper bound for it. Formally, CVaR equals the average of some percentage of the worst-case loss scenarios.

The interest in CVaR is based on two key advantages of CVaR over Value-at-Risk (VaR). First, CVaR, is informative about the tail end of the loss distribution than VaR. Second, the CVaR satisfies the four coherence axioms of Artzner, Delbaen, Eber and Heath (1999) whereas VaR fails the subadditivity requirement. Portfolio optimization with CVaR as risk measure was first studied by Rockafellar and Uryasev (2000), who showed that empirical CVaR minimization, can be formulated by a linear program.

The remainder of this paper is organized as follows. The next section briefly exposes some approaches concerning the use of CVaR in the portfolio optimization. Section 3 presents asset Allocation models to be resolved. The used data and estimation procedure are presented in section 4. Finally the results are discussed in section 5.

The advantages of $\mathrm{CVaR}$ over certain risk measures have led to the development of an extensive literature that explores the use $\mathrm{CVaR}$ in portfolio optimization. For example, portfolio optimization with CVaR as a risk measure is first studied by Rockafellar and Uryasev (2000), who show that empirical CVaR minimization can be formulated as a linear program. Rockafellar and Uryasev (2000) compare portfolios with minimum variance and CVaR given an expected return constraint. Krokhmal, Uryasev and Palmquist (2002) characterize portfolios with maximum expected return for various $\mathrm{CVaR}$ constraints with various confidence levels. They performed case study for the portfolio of S\&P100 Stocks. Their results show that the optimization algorithm which is based on linear programming technics is very stable and efficient.

Agarwal and Naik (2004) and Bertsimas, Lauprete and Samarov (2004) compare portfolios on the mean-variance and mean-CVaR boundaries. They show that mean-CVaR, unlike mean-VaR can be solved efficiently as a convex optimization problem, while the sample mean-shortfall portfolio optimization problem can be solved very efficiently as a linear optimization problem and they provide empirical evidence in asset allocation.

Alexander and Baptista (2004) provide an example with uniform distributions to illustrate that a CVaR constraint may be a more effective risk management tool than a VaR constraint. However, they do not characterize the constrained boundaries when security returns are assumed to have a discrete distribution with finitely many jump points.

Contrary to Sentana (2003) and Alexander and Baptista (2004) who examined the impact of VaR and CVaR constraints in the mean-variance model by assuming that security returns have an elliptical distribution, Alexander, Baptista and Yan (2007) explore the impact of adding either a VaR or a CVaR constraint to the mean-variance model, without making any distributional assumption. Alexander, Baptista and Yan (2007) showed that a CVaR constraint is more effective than a VaR constraint to curtail large losses in the mean-variance model, because the impact of adding either a VaR or a CVaR constraint to the mean-variance model when security returns are assumed to have a discrete distribution with finitely many jump points.

Our work differs from this literature in the fact that we compare the performance of optimal portfolios resulting from the mean-variance and mean-CVaR by using a dynamic trading strategy model controlling for the market conditions. Furthermore, frequently the return of asset are assumed to be normally or log-normally distributed. But the normal distribution is inadequate for describing the probability of extreme returns as usually encountered in practice. Our purpose is to investigate the effect of asymmetry and the fat tails on the solution of the resulting optimal portfolios.

\section{Asset Allocation Models}

We consider a multi-period model with $\mathrm{n}$ securities. At the beginning of the period, the portfolio manager can invest the money available in any of the $\mathrm{n}$ asset classes. The manager is not allowed to hold short positions. The objective of the portfolio manager is to minimize the risk of the portfolio subject to desired expected return accounting for transaction costs.

\subsection{Optimization of the Mean-CVaR Portfolio}

In this section we adopt the approach developed by Rockafellar and Uyasev (2000). Their method consists in solving the optimization of $\mathrm{CVaR}$ portfolios problem with a linear programming of a smooth and convex function. 
Let $f(x, y)$ be the returns associated with the decision vector $x$, itself chosen from a certain subset $X$ of $R^{n}$ and the random vector $\mathrm{y}$ of $\mathrm{R}^{\mathrm{m}}$. Then, the vector $\mathrm{x}$ can be interpreted as representing a portfolio, with $\mathrm{X}$ as the set of obtainable portfolios (subject to various constraints). The underlying probability distribution of $y$, represented by the density function $\mathrm{p}(\mathrm{y})$, and the probability of $\mathrm{f}(\mathrm{x}, \mathrm{y})$, not exceeding some threshold $\alpha$, is given by:

$$
\psi(x, \alpha)=\int_{f(x, y) \leq \alpha} p(y) d y
$$

Where $\psi$, as a function of $\alpha$ for fixed $x$, is the cumulative distribution function for the loss associated with portfolio $\mathrm{x} . \psi(\mathrm{x}, \alpha)$ is non-decreasing and continuous with respect to $\alpha$ and, as with $\mathrm{p}(\mathrm{y})$, is also continuous.

The VaR and CVaR values can be denoted as:

$$
\alpha_{\beta}(\mathrm{x})=\min \{\alpha \in \mathrm{R}: \psi(\mathrm{x}, \alpha) \geq \beta\},
$$

and

$$
\Phi_{\beta}(x)=(1-\beta)^{-1} \int_{f(x, y) \geq \alpha_{\beta}} f(x, y) p(y) d y
$$

In equation(3), the probability that $f(x, y) \geq \alpha_{\beta}$, is therefore equal to $1-\beta$. Thus, $\Phi_{\beta}(x)$ comes out as the conditional expectation of the loss associated with $\mathrm{x}$ relative to that loss being $\alpha_{\beta}(\mathrm{x})$ or greater.

The CVaR function in Equation (3) is difficult to handle, as it is a function of the VaR function. Using $\Phi_{\beta}(\mathrm{x})$ for the optimization of CVaR implies that VaR would have to be first calculated. The primary contribution by Rockafellar and Uryasev (1999) was the derivation of a CVaR function that was independent of the VaR function, making the optimization process much less complicated. Their function is given by:

$$
F_{\beta}(x, \alpha)=\alpha+(1-\beta)^{-1} \int_{y \in I^{m}}[f(x, y)-\alpha]^{+} p(y) d(y)
$$

Furthermore, the integral in the definition (4) of $\mathrm{F}_{\beta}(\mathrm{x}, \alpha)$ can be approximated in various ways. For example, this can be done by sampling the probability distribution of $y$ according to its density $\mathrm{p}(\mathrm{y})$. The corresponding approximation to $\mathrm{F}_{\beta}(\mathrm{x}, \alpha)$ is given by:

$$
\widetilde{\mathrm{F}_{\beta}}(\mathrm{x}, \alpha)=\alpha+(1-\beta)^{-1} \sum_{\mathrm{j}=1}^{\mathrm{J}} \pi_{\mathrm{j}}[\mathrm{f}(\mathrm{x}, \mathrm{y})-\alpha]^{+}
$$

Where $[t]^{+}=\max (t, 0)$ and $\pi_{j}$ are probabilities of scenarios $y_{j}$

$F_{\beta}(x, \alpha)$ Can be used instead of the CVaR measure. It has been proved that the function $F_{\beta}(x, \alpha)$ is convex with respect to $\alpha$ and that minimizing this function gives the same result as minimizing $\Phi_{\beta}(\mathrm{x})$ (Uryasev and Rockafellar, 2000).

The expression $\widetilde{\mathrm{F}_{\beta}}(\mathrm{x}, \alpha)$ is convex and piecewise linear with respect to $\alpha$. Although it is not differentiable with respect to $\alpha$, it can readily be minimized, either by line search techniques or by representation in terms of an elementary linear programming problem.

We can write the CVaR optimization problem as follows:

$$
\min \Phi_{\beta}(\mathrm{x})=\min \mathrm{F}_{\beta}[\mathrm{x}, \alpha(\mathrm{x}, \beta)]=\min \mathrm{F}_{\beta}(\mathrm{x}, \alpha)
$$

Or

$$
\min \left\{\alpha+(1-\beta)^{-1} \sum_{j=1}^{\mathrm{J}} \pi_{\mathrm{j}}[\mathrm{f}(\mathrm{x}, \mathrm{y})-\alpha]^{+}\right\}
$$

This follows from the derivative of the function $\mathrm{F}_{\beta}(\mathrm{x}, \alpha)$ with respect to $\alpha$ equals (Note 2):

$$
\frac{\mathrm{d}\left(\mathrm{F}_{\beta}(\mathrm{x}, \alpha)\right)}{\mathrm{d} \alpha}=1+(1-\beta)^{-1}[\psi(\mathrm{x}, \alpha)-1]
$$

When this derivative (6) equals zero, it is possible to derive the VaR that minimizes the function $F_{\beta}(x, \alpha)$ with respect to $\alpha$. From this, it follows that the minimization of the function with respect to both variables $\mathrm{x}$ and $\alpha$ optimizes $\mathrm{CVaR}$, and at the same time, delivers $\mathrm{VaR}$. This simplification of the CVaR function makes the optimization problem much easier, as there is no need to calculate the portfolio's VaR (Note 3).

Uryasev and Rockafellar (2000) considered minimizing CVaR while requiring a minimum expected return. By considering different expected returns, we can generate an efficient frontier. Alternatively, we also can also maximize returns while not allowing large risks. By considering different levels of risks, we can generate the efficient frontier.

Denoting by $r_{i}$ the return on asseti, we take the random vector to be $r=\left(r_{1}, r_{2}, \ldots, r_{n}\right)$. The distribution of $r$ constitutes a joint distribution of of the various returns on and is independent on $\mathrm{x}$; it has density $\mathrm{p}(\mathrm{r})$. The return on 
a portfolio $\mathrm{x}$, is the sum of the returns on the individual assets in the portfolio, scaled by the proportion $\mathrm{x}_{\mathrm{i}}$. The loss, being the negative of this, is therefore given by:

$$
f(x, r)=-\left[x_{1} r_{1}+x_{2} r_{2}+\cdots+x_{n} r_{n}\right]=-x^{T} r
$$

The objective function on which we focus is given by:

$$
F_{\beta}(x, \alpha)=\alpha+(1-\beta)^{-1} \int_{y \in R^{m}}\left[-x^{T} r-\alpha\right]^{+} p(r) d(r)
$$

It is important to observe that, $\mathrm{F}_{\beta}(\mathrm{x}, \alpha)$ is convex as a function of $\mathrm{x}$ and $\alpha$, not just $\alpha$. Often, it is also differentiable in these variables. Such properties set the stage very attractively for implementation of the kinds of computational schemes suggested above (Rockafellar and Uryasev, 2000).

Let $\mu(\mathrm{x})$ and $\sigma(\mathrm{x})$ denote respectively the mean and variance of the returns associated with portfolio; in terms of the mean $m$ and the variance of $x$,

We have:

$$
\mu(\mathrm{x})=-\mathrm{x}^{\mathrm{T}} \mathrm{m} \text { and } \sigma^{2}(\mathrm{x})=\mathrm{x}^{\mathrm{T}} \mathrm{V} \mathrm{x}
$$

Before presenting the problem formulation, which optimizes the CVaR for a confidence level $\beta$ subject to expected return less the transaction cost constraint and some other constraints, Let us introduce here auxiliary variables $\mathrm{z}=\left(\mathrm{z}_{1}, \ldots, \mathrm{z}_{\mathrm{J}}\right)$ such that: $\mathrm{z}_{\mathrm{j}} \geq \mathrm{f}\left(\mathrm{x}, \mathrm{r}_{\mathrm{j}}\right)-\alpha$, and $\mathrm{z}_{\mathrm{j}} \geq 0$, for $\mathrm{j}=1, \ldots, \mathrm{J}$

The $\mathrm{CVaR}$ minimization problem is presented as:

$$
\min \alpha+(1-\beta)^{-1} \sum_{j=1}^{J} \pi_{j} z_{j}
$$

Subject to:

$$
\begin{gathered}
x^{T} r_{j}+\alpha+z_{j} \geq 0 \quad z_{j} \geq 0, \quad j=1, \ldots, J \\
\sum_{i=1}^{n} x_{i} E\left(r_{i}\right)-\sum_{i=1}^{n} T C\left(x_{i}\right) \geq r_{p}^{*} \\
x_{i} \geq 0 \quad i=1, \ldots, n \\
\sum_{i=1}^{n} x_{i}=1 \\
x_{i} \leq 0,25 \quad i=1, \ldots, n
\end{gathered}
$$

Where $x$ is a $n \times 1$ Colum vector of portfolio weights $x_{i}, E\left(r_{i}\right)$ is the expected return for security $i, r_{p}^{*}$ is the minimal expected return and TC represents the transaction Costs function. Here we assume that transaction costs are linear and proportional to the bought or sold securities (Note 4). When buying or selling asset $\mathrm{i}$, one pays c times the amount of the transaction $\left|x_{i}-x_{i}^{0}\right|$, where $x_{i}^{0}$ is the security's position in the initial portfolio.

According to that, $\operatorname{TC}\left(\mathrm{x}_{\mathrm{i}}\right)=\mathrm{c}\left|\mathrm{x}_{\mathrm{i}}-\mathrm{x}_{\mathrm{i}}^{0}\right|$

This equality can be formulated using the following set of linear constraints:

$$
\begin{gathered}
\operatorname{TC}\left(x_{i}\right)=c\left(u_{i}^{+}+u_{i}^{-}\right) \\
x_{i}-x_{i}^{0}=u_{i}^{+}-u_{i}^{-}, \quad u_{i}^{+} \geq 0, \quad u_{i}^{-} \geq 0, \quad i=1, \ldots, n
\end{gathered}
$$

The constraint (11) ensures that short selling is not permitted. The second restriction in (12) is intuitive, since it ensures that the portfolios weights sum to unity. And the final mutual constraint (13) ensures that no portfolio weight exceeds $25 \%$. This constraint ensures a degree of diversification in the optimal portfolio.

In this framework, it is important to compare this program with the traditional problem of asset allocation that minimizes the variance (Markowitz, 1952).

\subsection{Optimization of the Mean-Variance Portfolio}

Optimizing the mean-variance Portfolio simply requires minimizing the variance of the portfolio subject to a number of constraints. Mathematically, the minimizing problem can be stated as:

$$
\min x^{\mathrm{T}} \sum \mathrm{x}
$$


Subject to:

$$
\begin{gathered}
\sum_{i=1}^{n} x_{i} E\left(r_{i}\right)-\sum_{i=1}^{n} c\left(u_{i}^{+}+u_{i}^{-}\right) \geq r_{p}^{*} \\
x_{i}-x_{i}^{0}=u_{i}^{+}-u_{i}^{-}, i=1, \ldots, n \\
u_{i}^{+} \geq 0, u_{i}^{-} \geq 0, i=1, \ldots, n \\
x_{i} \geq 0 ; \text { for } i=0, \ldots, n \\
\sum_{i=0}^{n} x_{i}=1 \\
x_{i} \leq 0,25 \text { for } i=0, \ldots, n
\end{gathered}
$$

Where $\mathrm{x}$ is a $\mathrm{n} \times 1$ Colum vector of portfolio weights and $\sum$ is the $\mathrm{n} \times \mathrm{n}$ variance-covanriance matrix of returns.

\section{Data and Estimation Procedure}

To compare the two problems, we use daily data for 20 stocks of the SBF 250 index. We consider only stocks of the SBF250 index with available data (price and dividend) (Note 5) and those entered the index before the 1st of January 2005 and were conserved until 31/12/2009. To ensure diversification, we choose 20 stocks such as the different segments of the stock market are represented. For each stock, we calculate the logarithmic returns. Therefore we obtain a time series of daily returns for each month of the periods studied.

Table 1 provides summary statistics for the 20 stocks. It is clear that many stocks return distributions are asymmetrical. In addition, the kurtosis statistic shows that the return series generally exhibit leptokurtic behavior, that is, have fatter tails than the normal distribution (the kurtosis statistic is greater than 3). The Jarque bera test indicates that the return distributions for all securities are significantly far from being normal at $1 \%$ level which implies that the distribution of returns has much thicker tails than the normal distribution.

Table 1. Statistical characteristics of stocks

\begin{tabular}{lrrrrrrrr}
\hline & \multicolumn{1}{c}{ Mean } & Median & Maximum & Minimum & deviation & Skewness & Kurtosis & JB test \\
\hline AIR LIQUIDE & 0,031 & 0,020 & 9,5 & -9 & 1,76 & $-0,029^{*}$ & $7,075^{*}$ & $703^{*}$ \\
AXA & $-0,023$ & 0,000 & 19,3 & $-20,4$ & 3,25 & $0,407^{*}$ & $10,168^{*}$ & $2224^{*}$ \\
BIC & 0,009 & 0,03 & 12,5 & $-11,2$ & 1,81 & 0,013 & $9,569^{*}$ & $1855^{*}$ \\
CASINO & 0,033 & 0,000 & 11,2 & $-12,5$ & 1,89 & $-0,063$ & $10,318^{*}$ & $2278^{*}$ \\
CLUB MED & $\mathbf{- 0 , 0 9 6}$ & $-0,048$ & 14 & $-11,3$ & 2,46 & $0,321^{*}$ & $7,208^{*}$ & $782^{*}$ \\
DANONE & 0,013 & 0,000 & 8,3 & $-8,5$ & 1,74 & $-0,230^{*}$ & $5,917^{*}$ & $371^{*}$ \\
EIFFAGE & $-0,004$ & $-0,059$ & 13,8 & $-11,8$ & 2,47 & 0,116 & $6,429^{*}$ & $503^{*}$ \\
ESSILOR INTL. & 0,026 & 0,026 & 13,3 & $-8,8$ & 1,52 & $0,58^{*}$ & $12,181^{*}$ & $3599^{*}$ \\
ESSO & $-0,024$ & 0,000 & 7,9 & $-8,6$ & 1,87 & $-0,143^{* * *}$ & $5,341^{*}$ & $240^{*}$ \\
FAURECIA & $-0,093$ & $-0,095$ & 17,3 & $-15,6$ & 2,91 & $0,145^{* * *}$ & $7,76^{*}$ & $974^{*}$ \\
L'OREAL & 0,029 & 0,039 & 13,7 & $-9,4$ & 1,78 & $0,377^{*}$ & $9,431^{*}$ & $1810^{*}$ \\
LOCINDUS & $-0,052$ & 0,000 & 16,4 & $-12,1$ & 2,12 & $1,009^{*}$ & $13,87^{*}$ & $5204^{*}$ \\
PERNOD-RICARD & 0,021 & $-0,019$ & 10,7 & $-14,6$ & 2,11 & $-0,383^{*}$ & $11,069^{*}$ & $2835^{*}$ \\
PPR & 0,002 & 0,000 & 14,2 & $-13,7$ & 2,59 & $0,386^{*}$ & $8,089^{*}$ & $1134^{*}$ \\
SANOFI-AVENTIS & $-0,016$ & 0,000 & 13,7 & $-10,9$ & 1,84 & 0,098 & $9,950^{*}$ & $2072^{*}$ \\
SCHNEIDER ELEC & 0,024 & 0,063 & 15,2 & $-16,2$ & 2,53 & 0,066 & $8,469^{*}$ & $1281^{*}$ \\
THALES & 0,004 & 0,000 & 5,7 & $-6,4$ & 1,52 & 0,002 & $4,501^{*}$ & $96^{*}$ \\
UNIBAIL-ROD & $\mathbf{0 , 0 9 8}$ & 0,104 & 10,2 & -8 & 2,18 & $0,124^{* * *}$ & $4,403^{*}$ & $86^{*}$ \\
VALLOUREC & 0,047 & 0,058 & 11,2 & $-17,5$ & 3,14 & $-0,323^{*}$ & $4,961^{*}$ & $182^{*}$ \\
VIVENDI & $-0,003$ & 0,000 & 13,5 & $-9,1$ & 1,73 & $0,287^{*}$ & $9,085^{*}$ & $1575^{*}$ \\
\hline
\end{tabular}


In this study we propose the dynamic allocation strategy where the simulations are carried through 2 steps. In the first step we estimate of the optimal portfolio weights using the data in the analysis window. Here an estimation window of one year is employed to estimate the mean return, the variance-covariance matrix and the CVaR. In the second stage, the calculation of the optimal portfolio performances over the detention period on a monthly basis. For example daily returns from $1^{\text {st }}$ of January 2005 to 31 st of January 2005 are used to calculate the mean return, the variance and covariance. Then these estimates are used as input to find the optimal portfolio weights for $1^{\text {st }}$ of January 2006. The resultant weights are used to calculate the performance over January 2006. This optimization process is repeated for each month until December 2009, yielding a total of 48 observations for the optimal portfolio's realized return and risk for each allocation model

\section{Optimal Portfolios Performance Using the Historical Distribution}

In a first scenario, we assume that the future distribution of the returns fully resembles the historical one. We work directly with a finite sample of return observations without assuming any specific distribution for returns. In that case, the full available set of historical returns is used as the scenario set over which mean returns, variance-covariance matrix and $\mathrm{CVaR}$ are estimated.

This section describes the dynamic asset allocation using the MV and MCVaR strategies where the parameters (ex. Expected returns, variances, $\mathrm{CVaR}$ ) are estimated from the historical returns. The two optimization processes use the same inputs. Here we estimate the CVaR with a confidence level of $95 \%$ and we use level of expected return accounting for transaction costs, $\mathrm{r}_{\mathrm{p}}^{*}=0 \%$ and we consider three different levels of transaction costs $(\mathrm{c}=0 \%$, $\mathrm{c}=0.15 \%$ and $\mathrm{c}=0.25 \%$ ).

Figure 1 displays the time series of monthly returns of the optimal portfolios under the MV and MCVaR approaches. The monthly returns of the optimal portfolio are computed by compounding daily returns within a month.
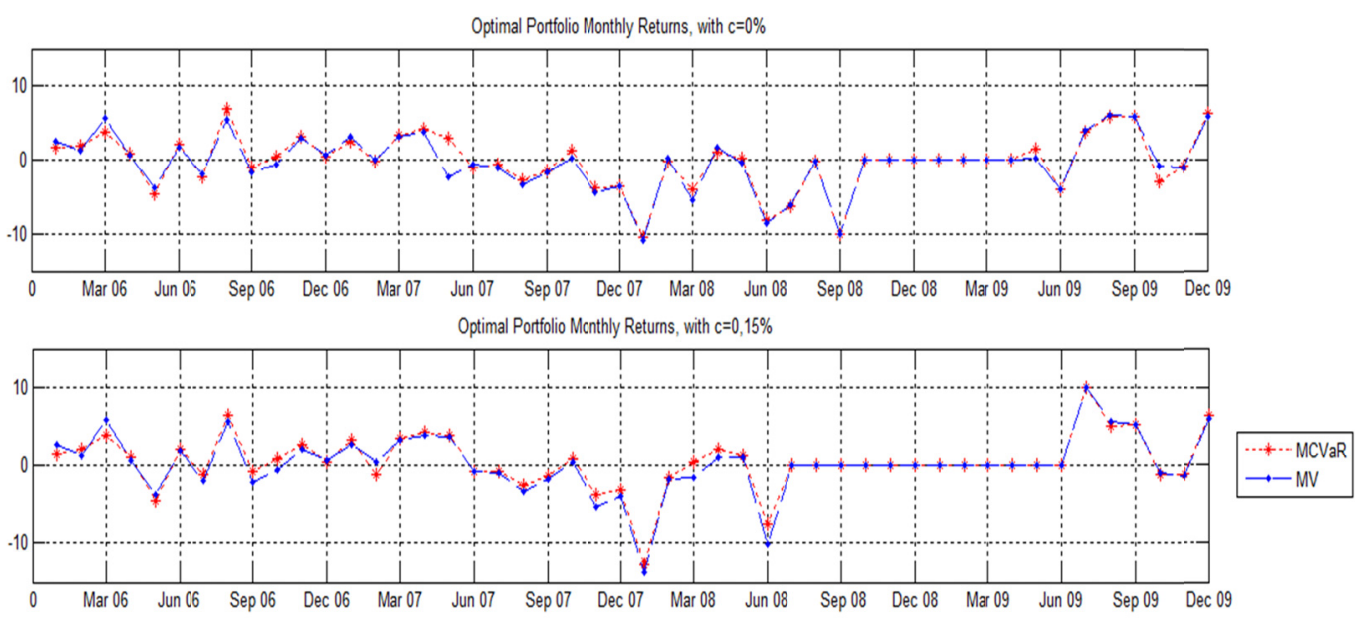

Optimal Portiolio Monthly Returns, with $c=0,25 \%$

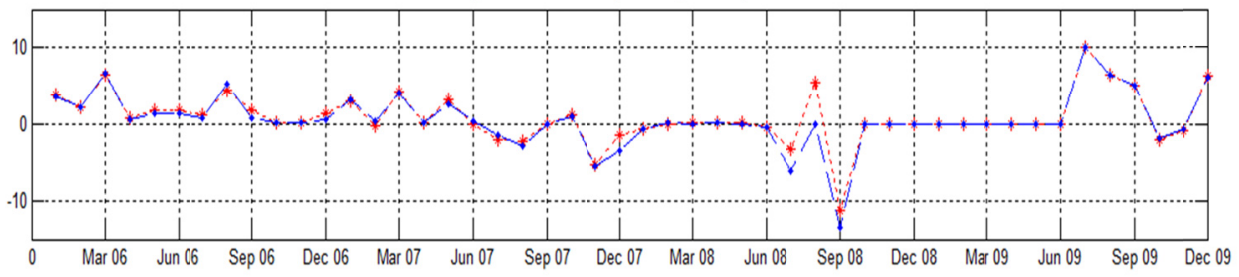

Figure 1. Monthly returns of the optimal portfolio under the MCVaR and MV approaches

Figure 1 clearly demonstrates that for almost months over the period 2006-2009, the CVaR framework performs better than the MV framework in terms of the realized monthly returns. The differences seen in the figure 1 are due to the departure from normality of returns. We notice here that there is a significant time-series variation of the monthly returns, requiring an analysis of the sensitivity of optimal performance to the market conditions. 
The time series of returns are summarized in Table 2, which also compares the relative performance of the MV and MCVaR optimal portfolios in terms of the monthly geometric mean returns, the mean Sharpe ratio and mean risk adjusted return of the optimal portfolios (return to CVaR ratio) over the whole period and the four one-year sub-periods. The sub-periods analysis enables to examine the implications of different data characteristics produced by different market conditions. These periods were chosen to capture both positive and negative financial market episodes; especially 2006 represent a flourishing period, while 2008 is recession period.

Table 2. MCVaR versus MV: Sub-periods analysis

Panel A. transaction cost $c=0 \%$

\begin{tabular}{|c|c|c|c|c|c|}
\hline & 2006-2009 & 2006 & 2007 & 2008 & 2009 \\
\hline \multicolumn{6}{|c|}{ Average returns } \\
\hline MCVaR & $-0,023$ & 0,091 & 0,003 & $-0,371$ & 0,156 \\
\hline MV & $-0,043$ & 0,084 & $-0,007$ & $-0,402$ & 0,161 \\
\hline Spread & 0,019 & 0,007 & 0,016 & 0,031 & $-0,005$ \\
\hline \multicolumn{6}{|c|}{ Sharpe Ratio } \\
\hline MCVaR & $-0,033$ & 0,030 & $-0,043$ & $-0,129$ & $-0,001$ \\
\hline MV & 0,007 & 0,013 & 0,112 & $-0,245$ & 0,124 \\
\hline Spread & $-0,040$ & 0,017 & $-0,156$ & 0,116 & $-0,125$ \\
\hline \multicolumn{6}{|c|}{ Risk adjusted return } \\
\hline MCVaR & 0,082 & 0,159 & 0,090 & 0,107 & 0,169 \\
\hline MV & 0,058 & 0,139 & 0,053 & $-0,120$ & 0,147 \\
\hline Spread & 0,024 & 0,021 & 0,037 & 0,227 & 0,021 \\
\hline \multicolumn{6}{|c|}{ Panel B. transaction cost $\mathrm{c}=0,15 \%$} \\
\hline & $2006-2009$ & 2006 & 2007 & 2008 & 2009 \\
\hline \multicolumn{6}{|c|}{ Average returns } \\
\hline MCVaR & 0,041 & 0,092 & 0,006 & -0272 & 0,324 \\
\hline MV & 0,012 & 0,076 & $-0,017$ & $-0,368$ & 0,323 \\
\hline spread & 0,029 & 0,015 & 0,024 & 0,096 & 0,001 \\
\hline \multicolumn{6}{|c|}{ Sharpe Ratio } \\
\hline MCVaR & 0,000 & 0,025 & $-0,025$ & 0,019 & $-0,016$ \\
\hline MV & 0,014 & 0,015 & 0,021 & 0,028 & $-0,015$ \\
\hline spread & $-0,014$ & 0,010 & $-0,047$ & $-0,008$ & $-0,001$ \\
\hline \multicolumn{6}{|c|}{ Risk adjusted return } \\
\hline MCVaR & 0,124 & 0,151 & 0,111 & $-0,075$ & 0,280 \\
\hline MV & 0,121 & 0,192 & 0,113 & $-0,123$ & 0,260 \\
\hline spread & 0,003 & $-0,041$ & $-0,002$ & 0,048 & 0,020 \\
\hline \multicolumn{6}{|c|}{ Panel B. transaction cost $c=0,25 \%$} \\
\hline & $2006-2009$ & 2006 & 2007 & 2008 & 2009 \\
\hline \multicolumn{6}{|c|}{ Average returns } \\
\hline MCVaR & 0,079 & 0,171 & $-0,006$ & $-0,101$ & 0,331 \\
\hline MV & 0,045 & 0,154 & $-0,020$ & $-0,204$ & 0,329 \\
\hline spread & 0,034 & 0,017 & 0,015 & 0,103 & 0,002 \\
\hline \multicolumn{6}{|c|}{ Sharpe Ratio } \\
\hline MCVaR & 0,120 & 0,294 & $-0,110$ & 0,286 & $-0,015$ \\
\hline MV & $-0,148$ & 0,268 & $-0,229$ & $-0,679$ & $-0,020$ \\
\hline spread & 0,268 & 0,025 & 0,119 & 0,965 & 0,005 \\
\hline \multicolumn{6}{|c|}{ Risk adjusted return } \\
\hline MCVaR & 0,119 & 0,204 & 0,068 & $-0,027$ & 0,273 \\
\hline MV & 0,106 & 0,201 & 0,059 & $-0,063$ & 0,241 \\
\hline Spread & 0,013 & 0,003 & 0,009 & 0,036 & 0,032 \\
\hline
\end{tabular}


Note: this table presents the average monthly returns expressed in percentage, average Sharpe ratio, average risk adjusted return of the optimal portfolios under the MV and MCVaR methods for the period 2006-2009 and for 4 one-year periods from 2006 to 2009. The results are based on monthly returns over the sample period. Panel A, B, C corresponds to transaction cost of $0 \%, 0,15 \%$ and $0,25 \%$ respectively. To be consistent with the standard measures of risk, we multiplied CVaRs by -1 (because CVaR is computed based on the negative tail of the return distribution, the original CVaR measures are negative).

Analyzing the performance of the optimal portfolios over the period as a whole, we can see that the MCVaR allocation model performs better in terms of mean return and mean adjusted risk return and unsurprisingly worse in terms of the Sharpe ratio than the MV allocation model. This result holds for the three considered transaction costs levels. The thorough examination of the optimal portfolio performance over the sub-periods shows that MCVaR optimal portfolio doesn't not perform the MV in 2006 in terms and that the discrepancies in rates of return between the two models (financial crisis) in 2008 are the most significant whereas the discrepancies in 2006, 2007 and 2009 are less significant. This result can explained by the fact that $\mathrm{CVaR}$ risk measure works better during bad states of the financial market and the main reason behind this, is that the financial crisis has put more weights to the left tail and therefore their impact on the MCVaR solution is higher. So the Market conditions appear to have a somewhat effect on the performance of the CVaR measure.

Another important finding from Table 2 demonstrates that decreasing the transaction costs clearly brings the two allocation frameworks much closer to each other; the difference between the MV and MCVaR approaches is more significant in presence of transaction. There is a difference of 2,5\% for a transaction cost of $0 \%$ and they increase to $3,2 \%$ for a transaction cost of $0,25 \%$ which means that since transaction costs are incorporated into the optimization problem, they also affect the choice of stocks but the MCVaR remains more performant than the MV model.

For robustness test, we repeat the same test but using two different confidence levels for the CVaR calculation $(\alpha=$ $90 \%$ and $\alpha=99 \%$ ) (Note 6). We find that decreasing $\alpha$ reduces the discrepancies between MCVaR and MV solutions whereas increasing the confidence level increases it. A high $\alpha$ means that only the extreme left part of the return distribution contributes to CVaR calculation and this is where the difference between the normal and non-normal distributions are most noticeable. When $\alpha$ decreases a larger portion of the distribution is involved in the calculation of $\mathrm{CVaR}$ and the portion where the departure from normality is largest, the extreme tail, has a lower influence on the final result.

\section{Optimal Portfolios Performance Using Simulated Distributions: The Impact of Skewness and Fat Tails on the Asset Allocation}

To measure the implications of non-normal characteristics of the assets, we use different scenarios to estimate the variance-covariance matrix and the CVaR measure. In Scenarios 2-6, we generate hypothetical probability distributions for the underlying assets with known parameters (Note 7). Scenario 2 assumes that the returns are given by the multivariate normal distribution which is possibly the easiest distribution to implement and where the parameters needed to be calculated are the mean returns and the variance-covariance matrix of the assets considered. Scenarios 3 and 4 involved Student-t with degree of freedom 3 and 4 respectively. Scenarios 5 and 6 involved a skewed fat tailed return distribution with degree of freedom 3 and 4 respectively. These distributions are chosen as they have the ability to control the characteristics of the empirical distribution. The Student-t distribution has heavy tails and non-zero tail dependence whereas the skewed student-t gives more control on the possible asymmetric on the positive and negative sides of return (Note 8).

In the remainder of this study we consider that there is no transaction costs, the expected return that should be achieved must be greater or equal to zero $0 \%$ and the confidence level used to estimate the CVaR is $95 \%$.

Table 3 compares the performance of optimal portfolios resulting from MV and MCVaR optimization models for the normal distribution, symmetric fat tailed distribution (Student- $t$ ), and the skewed fat tailed distribution. 
Table 3. The spread of performance between MV and MCVaR optimal portfolios for normal, student-t and skewed student-t distributions

\begin{tabular}{lrrrrr}
\hline & $\begin{array}{l}\text { normal } \\
\text { distribution }\end{array}$ & $\begin{array}{r}\text { student } \\
\text { distribution } \\
(\mathrm{v}=3)\end{array}$ & $\begin{array}{r}\text { student } \\
\text { distribution } \\
(\mathrm{v}=4)\end{array}$ & $\begin{array}{r}\text { skewed } \\
\text { distribution } \\
(\mathrm{v}=3)\end{array}$ & $\begin{array}{r}\text { skewed } \\
\text { distribution } \\
(\mathrm{v}=4)\end{array}$ \\
\hline Average returns & & & & & \\
\hline MCVaR & $-0,006$ & 0,033 & 0,010 & $-0,030$ & 0,048 \\
MV & 0,006 & 0,013 & 0,005 & $-0,031$ & 0,044 \\
spread & $-0,012$ & 0,020 & 0,006 & 0,001 & 0,004 \\
\hline Sharpe Ratio & & & & & \\
\hline MCVaR & $-0,012$ & $-0,057$ & 3,483 & $-0,404$ & 0,760 \\
MV & $-0,017$ & $-0,078$ & 2,664 & $-0,007$ & 0,590 \\
spread & 0,005 & 0,021 & 0,819 & $-0,397$ & 0,170 \\
\hline Risk adjusted return & & & & \\
\hline MCVaR & 0,096 & 0,088 & 0,123 & 0,112 & 0,136 \\
MV & 0,109 & 0,070 & 0,108 & 0,101 & 0,128 \\
spread & $-0,013$ & 0,018 & 0,015 & 0,011 & 0,008 \\
\hline
\end{tabular}

Note: this table presents the mean monthly returns expressed in percentage, the average Sharpe ration and the average Risk adjusted return of the optimal portfolio resulting from the MV and MCVaR optimization models. The results are based on the sample period January 2006-December 2009 and using generated data involving normal distribution, student-t distribution with $v=3 ; 4$ and Skewed Student-t distribution with $v=3 ; 4$

Comparing the performance of the optimal portfolios resulting from MV and MCVaR optimization models for different distributions we can see, that the variance is better risk measure than the CVaR when returns are normally distributed whereas the $\mathrm{CVaR}$ do better otherwise ( non-normal distributions).

For the non-normal distributions, table 3 shows that the superiority of $\mathrm{CVaR}$ over the variance is slightly more significant in the case of the Student- $t$ than in the case of skewned Student-t. The discrepancy of mean return ranges from $0.02 \%$ to $0.006 \%$ for Student-t density whereas it ranges from $0.001 \%$ to $0.004 \%$ for the skewed Student-t distribution. The same result holds on when performance is expressed in terms of the average return to CVaR ratio.

Table 3 shows also that in terms of risk adjusted return, the CVaR optimal turns out less performant when we increase the degree of freedom for the Student-t and Skewed Student-t density. The main reason is that when we increase the degree of freedom, the density is closer to the normal distribution.

Overall we can conclude that the MCVaR performance is most significant in the case of Student-t distribution with $\mathrm{v}=3$ and is less significant in the case of normal distribution. Therefore including the skewness and kurtosis into the asset allocation problem improves the performance in terms of average return and the average return-to-CVaR ratio but not in terms of Sharpe ratio. The information about both skewness and kurtosis can significantly impact the optimal allocations in the MCVaR optimization though the impact of the fat tails is more important.

\section{Conclusion}

In this study we examined the relevance of the MCVaR model and we explored the impact of non-normal features on its performance. In a first time we used the empirical distribution to compare the performance of this model with the performance the tradition MV model; The CVaR optimal portfolio seems to perform better in term of mean returns and the return-to-CVaR ratio but not in terms of the Sharpe ratio. This result holds on even when we consider the transaction costs and regardless the market conditions. An examination of the optimal performance over different market conditions shows that the performance of the CVaR model is more significant in bad states (financial crisis) of the financial market, periods when the departure from normality is generally more noticeable.

To measure the implications of non-normal characteristics of the assets, we generated normal distribution, fat tailed distributions and the Skewed Student-t distribution with two different degree of freedom, we find both skewness and kurtosis affect the MCVaR optimization and lead to substantially different allocations than do the traditional MV 
optimizations. The results show that accounting for skewness and more visibly for kurtosis improves the performance of the MCVaR optimal portfolio in terms of Mean return and risk adjusted return.

\section{References}

Acerbi, C., \& Tasche, D. (2002). On the coherence of expected shortfall. Journal of Banking and Finance, 26(7), 1487-1503. http://dx.doi.org/10.1016/S0378-4266(02)00283-2

Agrawal, V., \& N.Y., Naik. (2004). Risks and Portfolio Decisions Involving Hedge Funds. Review of financial studies, 17(1), 63-98. http://dx.doi.org/10.1093/rfs/hhg044

Alexander, G.J., \& Baptista, A.M. (2004). A comparison of VaR and CVaR constraints on portfolio selection with the mean-variance model. Management Science, 50(9), 1261-1273. http://dx.doi.org/10.1287/mnsc.1040.0201

Alexander, G.J., Baptista, A.M., \& Yan, S. (2007). Mean-Variance Portfolio Selection with 'At-Risk' Constraints and Discrete Distributions. Journal of banking and Finance, 31(12), 3761-3781. http://dx.doi.org/10.1016/j.jbankfin.2007.01.019

Alexander, S., Coleman, T., \& Li, Y. (2006). Minimizing CVaR and VaR for a portfolio of derivatives. Journal of Banking and Finance, 30(2006), 583-605. http://dx.doi.org/10.1016/j.jbankfin.2005.04.012

Andersson, F., Mauser, H., Rosen, D., \& Uryasev, S. (2001). Credit risk optimization with conditional Value-at-Risk criterion. Mathematical Programming, Series B, 273-291. http://dx.doi.org/10.1007/PL00011399

Artzner, P., Delbaen, F., Eber, J.M., \& Heath, D. (1999). Coherent measures of risk. Mathematical Finance, 9(3), 203-228. http://dx.doi.org/10.1111/1467-9965.00068

Biglova, A., Ortobelli, S., Rachev, S.T., \& Stoyanov, S. (2004). Different Approaches to Risk Estimation in Portfolio Theory. The Journal of Portfolio Management, 31(1), 103-112. http://dx.doi.org/10.3905/jpm.2004.443328

Bertsimas, D., Lauprete, G., \& Samarov, A. (2004). Shortfall as a risk measure: Properties and optimization. Journal of Economic Dynamics and Control, 28(7), 1353-1381. http://dx.doi.org/10.1016/S0165-1889(03)00109-X

Campbell, R., Huismann, K., \& Koedijk, K. (2001). Optimal portfolio selection in portfolio in value at risk framework. Journal of Banking and Finance, 25(9), 1798-1804. http://dx.doi.org/10.1016/S0378-4266(00)00160-6

Duffie, D., \& Pan, J. (1997). An overview of value at risk. Journal of Derivatives, 4(3), 7-49. Retrieved from http://www.iijournals.com/doi/abs/10.3905/jod.1997.407971?journalCode=jod

Fabozzi, F. J., Focardi, S.M., \& Kolm, P. N. (2006). Modelling of the equity market: From CAPM to cointegration. ed. Wiley.

Jorion, P. (2001). Value at Risk: The New Benchmark for Managing Financial Risk (second ed.). McGraw- Hill, New York.

Krokhmal, P., Palmquist, J., \& Uryasev, S. (2002). Portfolio Optimization with Conditional Value-At-Risk Objective and Constraints. Journal of Risk, 4(2), 11-27. Retrieved from http://www.ima.umn.edu/ santosa/wiki/images/4/4b/Cvar_2.pdf

Markowitz, H.M. (1952). Portfolio selection. Journal of Finance, 7(1), 77-91. http://dx.doi.org/10.2307/2975974

Markowitz, H.M. (1959). Portfolio Selection: Efficient Diversification of Investments. Yale University Press, New Haven, CT. Retrieved from http://cowles.econ.yale.edu/P/cm/m16/m16-all.pdf

Pflug, G.Ch. (2000). Some remarks on the value-at-risk and the conditional value-at-risk. In Uryasev, S. (Ed.), Probabilistic Constrained Optimization: Methodology and Applications. Kluwer Academic Publishers, Dordrecht. Retrieved from http://link.springer.com/chapter/10.1007/978-1-4757-3150-7_15

Rachev, S., Martin, D., Racheva-Yotova, B., \& Stoyanov, S. (2006). Stable ETL optimal portfolios and extreme risk management. Decisions in Banking and Finance. Springer. Retrieved from http://link.springer.com/chapter/10.1007/978-3-7908-2050-8_11

Rockafellar, R.T., \& Uryasev, S. (2000). Optimization of conditional value-at-risk. Journal of Risk, 2(3), 21-40. Retrieved

from http://www.pacca.info/public/files/docs/public/finance/Active\%20Risk\%20Management/Uryasev\%20Rockafell ar-\%20Optimization\%20CVaR.pdf 
Rockafellar, R.T., \& Uryasev, S. (2002). Conditional value-at-risk for general loss distributions. Journal of Banking and Finance, 26(7), 1443-1471. http://dx.doi.org/10.1016/S0378-4266(02)00271-6

Sentana, E. (2003). Mean-Variance Portfolio Allocation with a Value at Risk Constraint. Revista de Economía Financire, 1, 4-14. Retrieved from http://www.aefin.es/AEFIN_data/muestra.asp?art=A1-1.pdf

Xiong, J. X., \& Idzorek, T. (2011). The impact of Skewness and Fat Tails on the Asset Allocation Decision. Financial Analysts Journal, 67(2), 23-35. http://dx.doi.org/10.2469/faj.v67.n2.5

Yalçin, A., \& Yalcin, A. (2010). Optimal Portfolio Selection with a Shortfall Probability Constraint: Evidence from Alternative Distribution Functions. Journal of Financial Research, 33(1), 77-102. http://dx.doi.org/10.1111/j.1475-6803.2009.01263.x

\section{Notes}

Note 1. for a review see Duffie and Pan (1997)

Note 2. See the proof in Uryasev and Rockafellar (1999)

Note 3. A more detailed explanation of the derivation of the method is available in Uryasev and Rockafellar (2000, 2001)

Note 4. For more details on the transaction cost see, Fabozzi et al., 2006, chapter 3).

Note 5. Daily prices and dividends are gathered from Datastream.

Note 6. The results are not shown here, they are available upon request

Note 7. We used MATLAB to implement the distribution distributions; the code is presented in the appendix.

Note 8. For more details, see Emmanuel Jurczenko and Bertrand Maillet, "Multi-moment Asset Allocation and Pricing models", 2006 John Wiley \& Sons, Ltd, page 197 